\section{THE MYOCARDIAL PERFORMANCE DURING AND AFTER 72-HOURS OF THERAPEUTIC HYPOTHERMIA FOR PERINATAL ASPHYXIA IN TERM NEONATES}

doi:10.1136/archdischild-2012-302724.0293

${ }^{1} \mathrm{E}$ Nestaas, ${ }^{2,3} \mathrm{~J}$ Skranes, ${ }^{4,5} \mathrm{~A}$ Støylen, ${ }^{2,3} \mathrm{D}$ Fugelseth. 'Department of Paediatrics, Vestfold Hospital Trust, Tønsberg; 'Department of Neonatal Intensive Care, Os/o University Hospital, Ullevål; ${ }^{3}$ Faculty of Medicine, University of Oslo, Oslo; ${ }^{4}$ Department of Circulation and Medical Imaging, Faculty of Medicine, Norwegian University of Science and Technology; ${ }^{5}$ Department of Cardiology, St. Olavs Hospital, Trondheim, Norway

Background and Aims Therapeutic hypothermia after perinatal asphyxia reduces brain damage. The impact on the heart is poorly investigated. This study compare myocardial performance (MP) by ultrasound (Strain and Strain-Rate by tissue Doppler) in cooled asphyxiated term neonates (HT), asphyxiated term neonates treated with normothermia (NT) and non-asphyxiated term neonates (CTR).

Methods MP were compared in $44 \mathrm{HT}$ during and after cooling, 20 NT and 48 CTR.

Results The HT-group was more severely asphyxiated than the NTgroup (pH 7.07 (7.02, 7.12) (mean (95\%CI)) vs. 7.21 (7.14, 7.30), BaseExcess $-16.11 \mathrm{mmol} / \mathrm{L}(-17.8,-14.3)$ vs. $-9.3(-13.0,-5.6)(\mathrm{p}<0.05))$.
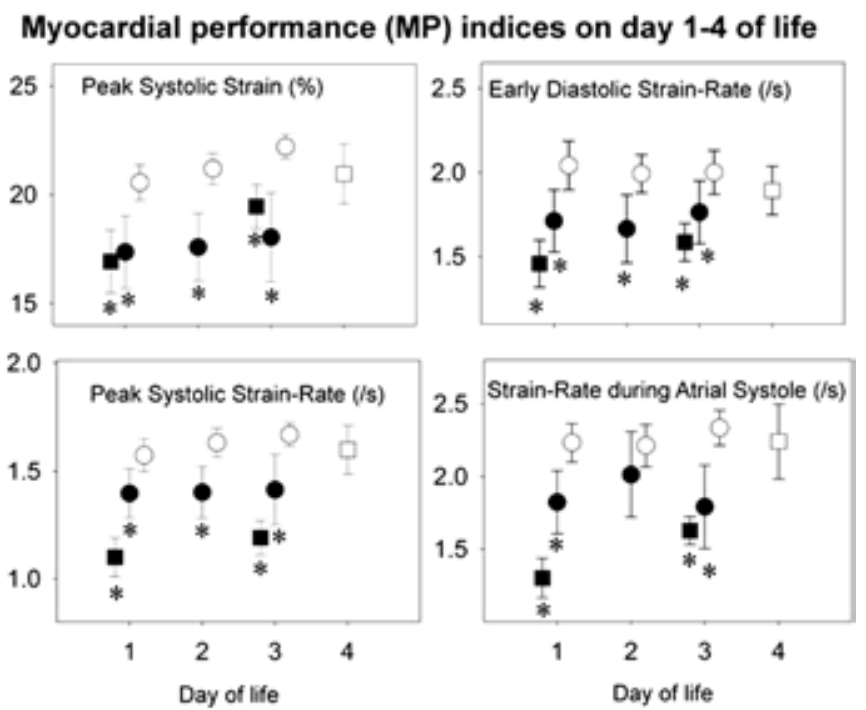

Absolute values (mean and $95 \%$ confidence intervals)

: Significantly different from the non-asphyxiated neonates $(p<0.05)$

Non-asphyxiated neonates (CTR group)

Asphyxiated normotherm neonates (NT group)

Asphyxiated neonates during therapeutic hypothermia (HT group)

Asphyxiated neonates after therapeutic hypothermia (HT group)

Abstract 293 Figure 1

On day 1-3, the MP was similar in the NT-group and HT-group during cooling, lower than in the CTR-group $(\mathrm{p}<0.05)$. The MP within each group was similar, except the CTR-group and HTgroup Peak Systolic Strain and HT-group Strain-Rate during the Atrial Systole $(\mathrm{p}<0.05)$.

After rewarming, the MP improved in the HT-group (day 4), approaching the MP in the CTR-group on day 3.

Conclusions Although the HT-group was more severely asphyxiated than the NT-group, the myocardial performance was similarly depressed on day 1-3. The myocardial performance in the HT-group improved after rewarming (day 4), approaching the level in the CTR-group on day 3 . Therapeutic hypothermia did not decrease the myocardial performance during treatment and might have had a positive impact after treatment.

\section{SUCCESSFUL IMPLEMENTATION OF HYPOTHERMIA TREATMENT IN SWEDEN - OUTCOME AT 24 MONTHS OF AGE IN HYPOTHERMIA-TREATED INFANTS WITH HIE}

doi:10.1136/archdischild-2012-302724.0294

K Robertson Grossmann, J Ragnö, B Vollmer, M Blennow, B Hallberg. Neonatology, Karolinska Institute, Karolinska University Hospital, Stockholm, Sweden

Background Hypoxic-ischemic encephalopathy (HIE) is associated with a high risk for subsequent neurological sequele. This study evaluates the implementation of HT in Sweden. Are national guidelines adhered to? Are outcome results comparable to those from previous controlled trials?

Methods Between 2007-2009, HT was available at 8 Swedish hospitals. Outcome data regarding all 187 infants who received HT during this period was collected and scored (normal/"motor delay"/ cerebral palsy (CP)/dead). Local guidelines for clinical follow-up differing from the national guidelines were also reviewed.

Results

Abstract 294 Table 1

\begin{tabular}{ll}
\hline Outcome & $\mathbf{N = 1 6 3 / 1 8 7}$ (24 children lost but alive) \\
\hline Normal & $109(58 \%)$ \\
"Motor delay" & $10(5 \%)$ \\
Cerebral palsy & $20(11 \%)$ \\
Dead & $24(13 \%)$ \\
\hline
\end{tabular}

Outcome data was available in $87 \%$ of treated infants. $13 \%$ had died, 74\% had been assessed between 15-36 months of age with a neurological exam and using scales for grading of severity of $\mathrm{CP}$, such as the Gross Motor Classification System. 35\% had been assessed according to national guidelines using Bayley III

Conclusions HT has been successfully implemented; however structured, comprehensive assessments according to national guidelines are not always carried out. In particular, only about $1 / 3$ of infants undergo a psychometric developmental assessment. The incidence of severe adverse neuromotor outcome is comparable to what was to be expected from earlier RCTs, showing that HT has a significant positive effect also outside of controlled trials.

\section{ROLE OF ANAPYREXIA IN PERINATAL ASPHYXIA IN TERM NEWBORNS: AN OPEN LABELED RANDOMIZED CONTROLLED TRIAL}

doi:10.1136/archdischild-2012-302724.0295

A Garg, A Saili, S Nangia. Department of Pediatrics, Kalawati Saran Children's Hospital, Delhi, India

Background and Aims To compare anapyrexia (a physiological decrease in body temperature in neonates in response to hypoxia) with normothermia (conventional management) with respect to immediate outcome and neuro-developmental outcome at 3 and 6 months of age in full term asphyxiated newborns.

Methods Fifty full term intramural neonates with severe birth asphyxia were randomized to Study (Anapyrexia) group and Control (Normothermia) group. Neonates in Anapyrexia group were allowed to remain at their own intrinsic temperature for a period of 72 hours. Neonates randomized to the Normothermia group had their temperature maintained between $36.5^{\circ} \mathrm{C}$ to $37.5^{\circ} \mathrm{C}$ with assistance from the servocontrolled radiant warmer. All neonates were managed according to standard management protocol of the unit. The subjects were monitored closely for any adverse effects, seizures, and development of HIE. The neurological examination was done at 6 hourly interval for the first 24 hours and then at 12 hourly interval. The surviving neonates were followed up at 3 months and 6 months of age. 